\title{
A Rethink of 10-2 Visual Fields in Early Glaucoma
}

\author{
Syed Shoeb Ahmad \\ Ajmal Khan Tibbiya College, Aligarh Muslim University, Aligarh, India
}

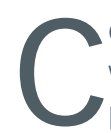
onventionally, the utilization of visual field programs for analysis of the central retina, such as the 10-2 test, has been confined to patients with advanced glaucoma. However, recently, a number of studies have shown that Humphrey visual field programs for testing the retinal periphery, such as the 30-2 or 24-2, often miss central defects. A subgroup of patients has been identified which present with central visual field defects during the early course of the disease while retaining normal peripheral visual fields. The diagnosis in these patients will be missed on 24-2 or 30-2 programs unless tested on strategies focused on the central retina. This is leading to a new awareness regarding performing of 10-2 tests early during the disease process. This review looks at the current role of this test in our clinical practice and whether a rethink is required regarding the application of the 10-2 test in the early stages of glaucoma.

DOI: https://doi.org/10.17925/USOR.2019.12.1.33

\section{Keywords}

Glaucoma, visual fields, macula

Disclosures: Syed Shoeb Ahmad has nothing to disclose in relation to this article.

Review Process: Double-blind peer review.

Compliance with Ethics: This study involves a review of the literature and did not involve any studies with human or animal subjects performed by any of the authors.

Authorship: All named authors meet the International Committee of Medical Journal Editors (ICMJE) criteria for authorship of this manuscript, take responsibility for the integrity of the work as a whole, and have given final approval to the version to be published.

Received: February 21, 2019

Accepted: March 29, 2019

Citation: US Ophthalmic Review. 2019;12(1):33-6

Corresponding Author: Syed Shoeb Ahmad, Ibn Sina Academy, Aligarh, 202001, India. E: syedshoebahmad @yahoo.com

Linkedln: https://in.linkedin.com/in/

syed-shoeb-ahmad-83b24685

Support: No funding was received in

the publication of this article.
Visual field (VF) analysis of the central retina is being touted by some clinicians as an indispensible investigation in all glaucoma patients, irrespective of the stage of the disease. ${ }^{1}$ The functional tests to investigate glaucoma patients analyze the VFs to look for loss of sensitivity. In early cases of glaucoma and glaucoma suspects the programs used on perimeters classically test the peripheral VF. This is based on the assumption that peripheral VF defects, such as the nasal step, are the earliest changes to develop in glaucoma. ${ }^{2,3}$ It was previously thought that macular function is affected at a stage late in the disease process. A central/temporal island of vision was presumed to be present until advanced disease set in. The so-called "tunnel vision" is the hallmark of advanced glaucoma. With such an understanding of the pathogenesis of glaucoma it was logical to explore the peripheral VF first. Thus, the 24-2 or 30-2 programs on the Humphrey visual field analyzer (HVFA) were deemed adequate to screen for glaucoma.

However, recently there have been a number of reports which document the involvement of macular fibers early in the disease process. Hood et al. have shown that early damage to the area near fixation can be very local and/or widespread. The latter defects can be very subtle owing to a loss of only a few decibels of sensitivity, and so their existence has been debated. ${ }^{1}$ But with the availability of structural studies there is irrefutable evidence of damage in the macular region of glaucoma patients. Hood and DeMoraes have cited the VF, optical coherence tomography (OCT) and anatomical basis for these assertions. ${ }^{1}$ OCT images from a large number of glaucoma patients show that macular retinal nerve fiber bundles do get damaged early in the development of glaucoma. ${ }^{4}$ Recently, OCTangiography (OCT-A) has also been utilized in cases of early glaucoma to confirm the loss of vessel density in the macula and resultant central 10-2 VF defects. ${ }^{5}$

A current hypothesis on structure-function relationship in early glaucoma is founded on the premise of a retinal ganglion cell (RGC) functional reserve or RGC redundancy. This hypothesis is consistent with reports suggesting that nearly half of RGCs and their axons could degenerate before any detectable loss of visual function on standard threshold VF testing. It is based on the assumption that the retina contains sufficient redundancy of RGCS, such that a subpopulation of them can retain normal sensitivity even when another subpopulation of RGCs is dysfunctional or lost. ${ }^{6}$ Nearly $30-50 \%$ of the RGCs are located in the macula. ${ }^{7}$ The axons of the RGCs form the retinal nerve fiber layer and exit through the optic nerve. Axons from the RGCs in the inferior region of the macula enter the disc in its most vulnerable part, namely, the temporal portion of the inferior quadrant. The temporal aspect of the disc is supposedly the site of the inferior- and superior-vulnerability zones (IVZ and SVZ), which show the largest loss in circumpapillary retinal nerve fiber layer. The portion of the IVZ which is associated with the macula (the "macular vulnerability zone" [MVZ]) is the region which gives rise to arcuate defects near fixation."

The macular region is unique in that it has the highest density of RGCs in the retina and is represented in over $60 \%$ of the visual cortex area. $24-2$ or $30-2$ perimetry is usually unable to detect paracentral 
scotomas at or near fixation due to the higher sensitivity at the macula. ${ }^{8}$ The macular area is vital for the acuity of vision required for many activities in daily life such as reading, driving, and face recognition. Thus, any pathology in this area is liable to affect the quality of life of the individual. ${ }^{9}$ Although central and paracentral scotomas usually develop in advanced stages of glaucoma, such defects are being increasingly identified during early disease. Some researchers advocate performing 10-2 VF testing for the central retina more routinely in order to diagnose glaucoma earlier, detect progression sooner, and minimize the risk of under- or overestimating the extent of glaucomatous VF damage in all stages of glaucoma. ${ }^{8}$ In view of the vital importance of the macula, patients with central defects require more aggressive treatment to preserve their visual function.

This review was undertaken to study whether the 10-2 VF tests in early stages of the disease may assist in the detection and monitoring of glaucoma; to perform a literature search on the subject; to understand the indications, limitations and developments being projected for the future regarding this test; and, based on this review, whether to recommend the 10-2 program to eye-care providers in early cases of glaucoma.

\section{The role of $10-2$ in glaucoma}

Glaucoma is currently defined as a multifactorial neurodegenerative disorder of the RGCs and the axons in the optic nerve. The diagnosis of glaucoma is heavily dependent on a number of structural and functional tests to assess the integrity of the neurosensory retina and the optic nerve during the development of glaucoma. The investigations to study the ocular structures include a number of techniques such as OCT, Heidelberg Retina Tomography and Retinal Nerve Fiber Layer Analyzer (GDx). Total macular thickness measured by some of these instruments is only a surrogate measure of the cellular loss in glaucoma. ${ }^{10}$ Newer techniques allow segmentation of the retinal layers. This has opened up to the realization that certain specific layers of the retina might be involved in the development of glaucoma. Segmentation also made these investigations more sensitive in the diagnosis of glaucoma, since a number of other diseases can affect this region. By studying the inner layers of the macula, known as the ganglion cell complex, clinicians are able to focus on those structures which are a prime target in glaucoma. These studies have shown that macular fibers are affected early in the development of glaucoma. ${ }^{11}$

The tests for analysis of functional deficits in glaucoma have evolved from manual techniques such as Bjerrum screen and Lister perimeter to the modern-day automated perimeters such as HVFA, Octopus perimeter, and those based on frequency doubling technology. The basic aim of these procedures is to map and analyze the VF. Conventionally, the VF refers to the total area in which objects can be seen in the side (peripheral) vision with the focus on a central point. The classical definition of Traquair described the VF as "an island of vision surrounded by a sea of blindness". The aim of perimetry, during the early stages of glaucoma, is to chart the boundaries of the island and monitor for progression, apart from assessment of the relative visual acuity of areas within those limits. ${ }^{12}$

In order to assess the contours of the island, the HVFA uses programs which cover a large part of the VF such as the 30-2 and 24-2 test patterns. Based on our assumption that the periphery of the island of vision is affected in early glaucoma, the above-mentioned are the two test patterns most commonly employed. As disease advances, the island shrinks to such an extent that only the macula survives and thus, peripheral tests become redundant. In such cases of advanced glaucoma the 10-2 program on the HVFA is used to analyze only the surviving central $10^{\circ}$ of the retina. ${ }^{13}$

Structural studies such as macular thickness mapping have shown significantly lower retinal thickness in early manifest glaucoma patients, compared to normal controls. OCT studies have also shown that retinal thickness in the macula is associated with a corresponding loss of central VF in early and moderate glaucoma. ${ }^{14}$ Functional assessment of this group of patients demonstrates apparently normal central VFs when tested on 30-2 programs. ${ }^{15}$ The programs to study the peripheral VF are based on a $6^{\circ}$ grid pattern. With such large grids, the macula is poorly represented on VF analysis. On a $24-2$ program, hardly 12 points are tested in the central $10^{\circ}$. since the use of 10-2 program has been commonly employed in patients with advanced glaucoma, individuals with solely central defects early in the course of disease will be missed out with the 24-2 or 30-2 tests. ${ }^{8}$ Supported by real-time evidence from OCT, OCT-A, and microperimetry, studies confirm macular damage during the early stages of glaucoma. ${ }^{5,11}$ Therefore, these patients will show anomalies on VF analysis only if the 10-2 tests, which use a finer $2^{\circ}$ grid, are utilized. This scenario could be so widespread that some studies report almost an equal occurrence of peripheral and central VF defects in the studied population. ${ }^{16}$ This significant development is leading to a paradigm shift in our understanding and investigation of glaucoma.

The macula lutea is a crucial part of the retina. Concerned with central vision, this area contains approximately $30-50 \%$ of all RGCS. ${ }^{17,18}$ It contains the target cells directly affected in glaucoma. ${ }^{11}$ The earliest mention of a paracentral scotoma occurring in glaucoma is attributed to Von Graefe. ${ }^{19}$ Later, it was Drance who elucidated these changes in his article published in the Investigative Ophthalmology Journal in 1969. According to him, the earliest field defects in glaucoma are paracentral scotomas in the Bjerrum area separated from the blind spot and later coalescing into an arcuate scotoma joining the blind spot. ${ }^{20}$ Paracentral scotomas are critical because any damage to this area severely affects the quality of life of the patient, with increased risk of falls, hip fractures, and mortality. ${ }^{21}$

Over the years, there have been only intermittent reports regarding macular involvement in early glaucomatous damage. Heijl and Lundqvist in their study to document the frequency distribution of earliest glaucomatous VF defects reported certain patients who had normal VFs on the 24-2 test but showed arcuate defects in the central $10^{\circ}$ on $10-2$ VFs. ${ }^{22}$ Hood et al. studied $V F$ defects in the central retina in patients who had apparently normal 24-2 test results. Sixteen eyes among all patients included in the study (27 eyes in total) had normal $24-2$ fields in the central $10^{\circ}$ but revealed defects on 10-2 VF tests. Out of these, 12 eyes had arcuate defects and 4 eyes had other defects in the macular area. The retinal nerve fiber layer (RNFL) defects were confirmed on OCT scans. ${ }^{23}$ Traynis et al. performed 10-2 and 24-2 VF assessments on 50 subjects diagnosed as glaucoma suspects or who showed signs of early glaucoma. They reported $53 \%$ of the hemifields as abnormal on $10-2$ fields compared to $59 \%$ on $24-2$ VFs. In that study, $16 \%$ of the hemifields that were normal on the $24-2$ VFs were abnormal on the 10-2 test. Consequently, the researchers concluded that the 24-2 test is not optimal for detecting early damage of the macula. ${ }^{16}$ In our own clinical practice, patients are assessed primarily on 24-2 tests if there are no features suggestive of macular involvement. However, with this awareness that 10-2 tests may show changes even in those patients who have no 
central defects on 24-2, we are slowly incorporating 10-2 tests in our practice. Presently, this approach is reserved for patients who have macular abnormalities on OCT, or unexplained symptoms.

Scheifer and colleagues in their study of mild to moderate glaucoma patients found over $50 \%$ of them having defects within the central $+/-3^{\circ} .{ }^{24}$ Langerhost also found 9\% of the hemifields classified as normal with the 30-2 being classified as abnormal on 10-2 testing. ${ }^{25}$ DeMoraes and colleagues have compared the prevalence of abnormal 24-2 versus 10-2 VF results based upon cluster criteria in glaucoma suspects, ocular hypertensives, and early glaucoma patients. In glaucoma suspects $39.5 \%$ eyes classified as normal on 24-2 were classified as abnormal on 10-2 VFs. Similarly, $35.4 \%$ eyes in ocular hypertensives and in glaucoma patients $61.5 \%$ eyes showed abnormal results on 10-2 tests despite normal 24-2 VFs. ${ }^{26}$ Deva et al. performed 24-2 VFs on 107 patients with newly diagnosed glaucoma and demonstrated $56 \%$ of them having an abnormality within $10^{\circ}$ of fixation. ${ }^{27}$

A number of alternative strategies have been suggested in order to incorporate central VF assessment in routine clinical practice when investigating patients with early glaucoma. One solution recommended to address the problem of missed central defects is to perform 10-2 VFs in all glaucoma patients. ${ }^{1}$ However, performing additional tests to the conventional 24-2 test is time consuming and tiring for patients. Consequently, the results of VF testing become less reliable with each extra test. ${ }^{9}$ Another limitation of $10-2$ testing is that progression is difficult to gauge since test patterns are not consistent and the number of 10-2 VFs is often small. ${ }^{21}$

Since combined peripheral and central VF testing may not be feasible due to limitations of time, staff and patient co-operation, some authors suggest performing the 10-2 tests at a separate sitting than the $24-2$ tests. ${ }^{1}$ Over a period of time this will provide the eye-care provider with a progressively larger databank of 10-2 results which can be used to assess and monitor the patients. Some other researchers have made certain recommendations for performing 10-2 tests in cases of early glaucoma. ${ }^{1,28}$ According to them this program should be considered in the following circumstances:

- There are any depressed points in the central $12^{\circ}$ less than $0.5 \%$ on $24-2$ or 30-2 VF testing.

- The paracentral defect is greatly depressed relative to the mean deviation (MD) on 24-2.

- There are any abnormal points in the central 12 points on $24-2$ testing that spatially correlate to thinning in the macular ganglion cell inner plexiform layer area.

- Patients with an abnormal region on RGC analysis of an OCT scan of the macula.

- An abnormal region in the temporal quadrant or MVZ on circumpapillary retinal nerve fiber layer analysis of the OCT scan of the disc.

- A visual acuity that cannot be corrected to 20/20.

- Complaints about glare or trouble reading that cannot be attributed to other causes.
As the $6^{\circ}$ grid of the 24-2 test does not adequately analyze the macula, a modified program to comprehensively assess the periphery as well as the central retina should be developed. However, development of newer programs is hampered as clinicians are reluctant to give up 24-2 testing because they have years of 24-2 data for their patients; they are accustomed to interpreting 24-2 reports and this test reveals arcuate scotomas better, which can be missed on 10-2 tests. In order to overcome these limitations, Ehrlich and colleagues suggested adding 4 to 16 test points to the conventional 24-2 test pattern. They reported significantly improved ability to detect macular defects with this strategy. ${ }^{9}$ Asaoka has proposed a novel "Lasso regression" method by which the 10-2 VF MD can be better predicted by incorporating data from 24-2 VFs. The proposed method improves the accuracy of progression estimates on 10-2 VF testing. This approach can help clinicians to better assess and predict visual function in the parafoveal area. ${ }^{2}$

Commercially, the closest solution to these problems is the G-program in the Octopus perimeter which has a modified $6^{\circ}$ grid capable of assessing for macular and perimacular damage. Carl Zeiss has also developed a 24-2 test (called the C24-2) augmented with additional points from the 10-2 test. A study did find substantial agreement between the cases detected by the C24-2 and 10-2 test patterns. There was also a very strong association between the pattern standard deviation (PSD) values from the two methods. ${ }^{29}$ These results give us hope of better test strategies to perform a rapid comprehensive analysis of the entire retina in glaucoma patients.

\section{Conclusion}

Sensitivity loss in the macular region was regarded as a late manifestation of glaucoma. Therefore, VF analysis of this region was only undertaken in advanced stages of the disease. However, as this review highlights, macular damage could occur at any stage of the disease. Since the 24-2 or 30-2 programs do not adequately test this vulnerable region, patients with damage in the central retina could be classified as normal. Therefore, tests to analyze the macular region such as the 10-2 on HVFA are presently recommended for all patients in whom 24-2 and/or 30-2 tests are indicated. ${ }^{1}$ The tests for peripheral damage as well as central loss should be incorporated in our clinical practice in order to test the entire retina adequately. In our opinion, anyone you would, or have done, a 24-2 VF on should have both a 24-2 and a 10-2 VF within the first two visits. However, we face challenges in the way of resources (such as the optometrists required to perform the perimetry and the machinery they use) and patient access to the clinic (such as the long distances they have to travel). Once newer tests, which would combine analysis of the macula and peripheral retina become more readily available, then they can be expeditiously used to our advantage. Such tests need to look into the challenges of performing the test in a reasonable time so as not to compromise the results from patient fatigue, should be user friendly, should be convenient to look for progression without the need to re-educate the practitioners and easily installed in current machines. Looking at the recommendations provided by the studies mentioned in this review there appears to be a need to rethink the indications for performing 10-2 VFs in early glaucoma. $\square$
1. Hood DC, De Moraes CG. Four questions for every clinician diagnosing and monitoring glaucoma. J Glaucoma. 2018;27:657-64.

2. Goldberg I. Optic disc and visual field changes in primary open angle glaucoma. Aust J Ophthalmol. 1981;9:223-9.

3. Werner B, Beraskow J. Peripheral nasal field defects in glaucoma. Ophthalmology. 1979;86:1875-8.
4. Arvanitaki V, Tsilimbaris MK, Pallikaris A, et al. Macular retinal and nerve fiber layer thickness in early glaucoma: clinical correlations. Middle East Afr J Ophthalmol. 2012;19:204-10.

5. Penteado RC, Zangwill LM, Daga FB, et al. Optical coherence tomography angiography macular vascular density measurements and the central 10-2 visual field in glaucoma J Glaucoma. 2018;27:481-9.
6. Ventura LM, Sorokac N, De Los Santos R, et al. The relationship between retinal ganglion cell function and retinal nerve fiber thickness in early glaucoma. Invest Ophthalmol Vis Sci. 2006;47:3904-11.

7. Mota M, Vaz FT, Ramalho M, et al. Macular thickness assessment in patients with glaucoma and its correlation with visual fields. J Curr Glaucoma Pract. 2016;10:85-90. 
8. Lifferth A, Fisher B, Stursma A et al. 10-2 visual field testing: A tool for all glaucoma stages. Review of optometry. 2017. Available at: www.reviewofoptometry.com/article/ ro0717-102-visual-field-testing-a-tool-for-all-glaucoma-stages (accessed March 28, 2019).

9. Ehrlich AC, Raza AS, Ritch R, et al. Modifying the conventional visua field test pattern to improve the detection of early glaucomatous defects in the central 10 $0^{\circ}$. Trans/ Vis Sci Technol. 2014:3:6.

10. Gupta D, Asrani S. Macular thickness analysis for glaucoma diagnosis and management. Taiwan J Ophthalmol. 2016;16:3-7.

11. Kim NR, Lee ES, Seong GJ, et al. Structure-Function relationship and diagnostic value of macular ganglion cell complex measurement using Fourier-Domain OCT in glaucoma. Invest Ophthalmol Vis Sci. 2010:51:4646-51

12. Grzybowski A. Harry Moss Traquair (1875-1954), Scottish Ophthalmologist and perimetrist. Acta Ophthalmol. 2009;87:455-9.

13. Zalta AH. Use of a central 10 degrees field and size $V$ stimulus to evaluate and monitor small central islands of vision in end stage glaucoma. Br J Ophthalmol. 1991;75:151-4.

14. Liu CH, Chang SHL, Wu SC. Regional relationship between macular retinal thickness and corresponding central visual field sensitivity in glaucoma patients. J Ophthalmol. 2017:2017:3720157.
15. Asaoka R. Mapping glaucoma patients $30-2$ and $10-2$ visual fields reveals clusters of test points damaged in the 10-2 grid that are not sampled in the sparse 30-2 grid. PLOS ONE. 2014;9:e98525.

16. Traynis I, De Moraes CG, Raza AS, et al. The prevalence and nature of early glaucomatous defects in the central $10^{\circ}$ of the visual field. JAMA Ophthalmol. 2014;132:291-7.

17. Hood DC, Raza AS, De Moraes CG, et al. Glaucomatous damage of the macula. Prog Retin Eye Res. 2013;32C:1-21.

18. Um TW, Sung KR, Wollstein G, et al. Asymmetry in hemifield macular thickness as an early indicator of glaucomatous change. Invest Ophthalmol Vis Sci. 2012;53:1139-44.

19. Von Graefe A. Beitrage zur Pathologie und Therapie des Glaukoms. Arch J Ophth. 1869;15:108.

20. Drance SM. The early field defects in glaucoma. Invest Ophthalmol. 1969;8:84-91.

21. Asaoka R. Measuring VF progression in the central $10^{\circ}$ using additional information from central 240 visual fields and "Lasso Regression". PLOS ONE. 8(8):e72199.

22. Heijl A, Lundqvist $L$. The frequency distribution of earliest glaucomatous visual field dfects documented by automatic perimetry. Acta Ophthalmol (Copenh). 1984;62:658-64.

23. Hood DC, Raza AS, De Moraes CG, et al. Initial arcuate defects within the central $10^{\circ}$ in glaucoma. Invest Ophthalmol Vis Sci.
2011;52:940-6.

24. Scheifer U, Papageorgiou E, Sample PA, et al. Spatial pattern of glaucomatous visual field loss obtained with regionally condensed stimulus arrangements. Invest Ophthalmol Vis S 2010;51:5685-9.

25. Langerhost CT, Carenini LL, Bakker $D$, et al. Measurements for description of early glaucomatous field defects. In: Wall M, Heijl A (eds). Perimetry Update 1996/1997. New York: Kugler Publications, 1997;67-73.

26. De Moraes CG, Hood DC, Thenappan A, et al. 24-2 visual fields miss central defects shown on 10-2 tests in glaucoma suspects, ocular hypertensives and early glaucoma. Ophthalmology. 2017;124:1449-56.

27. Deva NC, Insual E, Gamble G, et al. Risk factors for first presentation of glaucoma with significant visual field loss. Clin Exp Ophthalmol. 2008;36:217-21.

28. Park H, Hwang B, Shin $H$, et al. Clinical clues to predict the presence of parafoveal scotoma on Humphrey $10-2$ visua field using a Humphrey 24-2 visual field. Am J Ophthalmol. 2016;161:150-9

29. Wu Z, Madeiros FA, Weinreb RN, et al. Performance of the 10-2 and 24-2 visual field tests for detecting central visual field abnormalities in glaucoma. Am J Ophthalmol. 2018;196:10-17. 